\title{
Structural features of enzymatic hydrolysis lignin from the corn stalks and furfural residue
}

\author{
Fu Aixiao ${ }^{1}$, Li Zhui ${ }^{2}$, Lian En Xiao ${ }^{3}$, Deng Yilin ${ }^{4}$, Ma Yanli ${ }^{5,{ }^{*}}$ \\ College of Material Science and Engineering, Northeast Forestry University, China \\ myl219@126.com
}

\begin{abstract}
Keywords: structural features, lignin, corn stalks, furfural residue
Abstract. Structural features of enzymatic hydrolysis lignin from the corn stalks and furfural residue was compared with lignin extracted from paper-making black liquid (PBL). Lignin was characterized by FT-IR, GPC, ${ }^{13} \mathrm{C}$ - and ${ }^{1} \mathrm{H}$ - NMR analysis. Enzymatic hydrolysis lignin from the corn stalks (CS) had a $12.2 \%$ higher phenolic hydroxyl content and FR had a 4.74\% higher than that of PBL. FR had lower alcoholic droxyl content than that of CS and PBL. The Mw of CS was determined to be 5,520Da, which was 1.5 times of the Mw of PBL (3,660 Da).The Mn of both FR and PBL had similar values of about $4000 \mathrm{Da}$. the Mn of CS was only $1903 \mathrm{Da}$. The polydispersity index (Mw/Mn) of CS and FR were calculated to be 1.92 and 1.70, respectively. The lignin of CS and FR consisted of $\beta$-O-4units, the $\mathrm{C}-\gamma$ with $\alpha-\mathrm{C}=\mathrm{O}$ originated from side chain oxidation via hemolytic cleavage and condensation of $\beta-\beta$ structures during enzymatic hydrolysis process.
\end{abstract}

\section{Introduction}

Lignin is one of the main components of plants that accounts, and is the second most abundant polymeric material in nature next to cellulose. Lignin is known to bind physically/chemically to cellulose/ hemicellulose by covalent bonding such as benzyl-ether, benzyl-ester and phenyl-glycoside bonds, forming lignin-carbohydrate complexes (LCC) in plant cell walls [1]. In recent years, bioethanol has received great attention due to energy crisis and global warming issue [2]. A large number of studies propose the utilization of lignocellulosic biomass as a feedstock for producing fuel ethanol[3]. However, extraction methods of alkali lignin in existing separation process are not easy to apply into industrial-scale production due to the high cost or complex process. So an adequate separation method is necessary for alkali lignin from industry residue's future application. At same time, comparative analysis structures from different preparation process of alkali lignin is necessary for effective development of lignin composite materials.

The aim of this paper is to establish a system in separating lignin from bio-ethanol production residue, such as enzymatic hydrolysis lignin from the corn stalks and furfural residue. Extraction, a commonmethod already applied in industry, is adopted in our research and sodium hydroxide and ethanol are chosen as the solvents. The lignin products were further quantified and characterized by a comprehensive set of analytical methods, including FTIR, GPC, ${ }^{1} \mathrm{H}-\mathrm{NMR}$ and ${ }^{13} \mathrm{C}-\mathrm{NMR}$.

\section{Methods}

Chemicals. Air-dried enzymatic hydrolysis lignin from the corn stalks (CS) was obtained from a large-scale pilot plant where converting steam-exploded cornstalk into bio-ethanol with SSF method. Its lignin and ash content were determined to be $77.69 \%$ and $14.96 \%$, respectively Enzymatic hydrolysis lignin from the furfural residue was obtained from furfural production, its lignin and ash content were determined to be $65.28 \%$ and $9.96 \%$, and the rest component of above were remained carbohydrates. For comparative analysis, lignin extracted from paper-making black liquid (PBL) was used as a representative native lignin, extracted from straw, and served as a control. The other chemicals were pure grade reagents commercially available.

Preparation of enzymatic hydrolysis lignin. Air dried enzymatic hydrolysis furfural residue (FR) and corn stalk are ground to a fine powder (less than $0.64 \mathrm{~mm}$ particle size). The powder $(1.0 \mathrm{~g})$ was added to $34 \mathrm{~mL} 2 \% \mathrm{NaOH}$ solution was stirred at $80{ }^{\circ} \mathrm{C}$ for $4 \mathrm{~h}$ to extract lignin and then wash the extraction by distilled water until without chloride ion. 
Functional groups. The essential functional groups attached to the lignin, methoxyl and phenolic hydroxyl moieties, were determined by FT-IR and aminolysis methods, respectively.

Gel permeation chromatography (GPC). The lignin was acetylated with acetic anhydride/pyridine $(1: 1 \mathrm{v} / \mathrm{v})$ at $70^{\circ} \mathrm{C}$ for $6 \mathrm{~h}$, and the derivatized lignin was dissolved in tetrahydrofuran (THF) and analyzed by Aglient 1100 GPC equipped with 79911GF-083column (100 to 30,000, $25 \mu \mathrm{m}$,). To determine the molecular weight of the effluent, polystyrenes with a mass range between $1000 \mathrm{Da}$ and 680,000 Da were used to create a calibration curve.

Spectroscopic analysis (FT-IR, ${ }^{\mathbf{1}} \mathbf{H}$ - and ${ }^{\mathbf{1 3}} \mathbf{C}$-NMR). FT-IR spectra were recorded on a Nicolet 5500 spectrometer (Thermo Scientific, USA) operating in the wavelength range of $4000-400 \mathrm{~cm}^{-1}$ with a resolution of $16 \mathrm{~cm}^{-1}$. Each sample was coated by $\mathrm{KBr}$ and formed into a pellet for analysis. ${ }^{13} \mathrm{C}-\mathrm{NMR}$ and ${ }^{1} \mathrm{H}-\mathrm{NMR}$ spectra were obtained with a Bruker ultrashield 500 plus and 300 spectrometer (Bruker, Germany) operating at $25^{\circ} \mathrm{C}$, respectively, with $150 \mathrm{mg}$ of lignin sample dissolved in DMSO-d ${ }_{6}$.

\section{Results and discussion}

Functional groups. Table 1 shows the functional groups of the two enzymatic hydrolysis lignin samples, extracted with $\mathrm{NaOH}$ solution and purified by the Björkman procedure[3], respectively. For the phenolic $\mathrm{OH}$ content, enzymatic hydrolysis lignin from the corn stalks (CS) had a $12.2 \%$ higher phenolic hydroxyl content and FR had a $4.74 \%$ higher than that of PBL, which indicates that CS and FR consist of more free-phenolic moieties that are probably released by partial fragmentation of lignin during the enzymatic hydrolysis extraction. However, FR had a lower alcoholic droxyl content than that of CS and PBL. The reason that the lignin side chains were destroy by furfural production.

Table 1. Functional groups of lignin from the enzymatic hydrolysis corn stalks (EL), furfural residue FR) and pulping black liquor (PBL).

\begin{tabular}{lllll}
\hline \multicolumn{2}{c}{ Lignin } & CS & FR & \multicolumn{1}{c}{ PBL } \\
\hline $\begin{array}{c}\text { Functiona } \\
\begin{array}{c}1 \\
\text { group }(\%)\end{array}\end{array}$ & Ar-OH & 7.82 & 7.65 & 6.97 \\
\hline
\end{tabular}

Structural characterizations of CS and FR. The average molecular weights (Mw and Mn) and polydispersity index of both lignin samples were determined by GPC analysis, and the results are given in Table 2. The Mw of CS was determined to be 5,520Da, which was 1.5 times of the Mw of PBL $(3,660 \mathrm{Da})$. Together with the high content of phenolic $\mathrm{OH}$, this lower $\mathrm{Mw}$ value for $\mathrm{CS}$ is good evidence for lignin fragmentation by enzymatic hydrolysis. The higher Mw value for FR indicated that lignin condensation during hydrolysis. In contrast, the Mn of both FR and PBL had similar values of about $4000 \mathrm{Da}$. the Mn of CS was only $1903 \mathrm{Da}$. The polydispersity index (Mw/Mn) of CS and FR were calculated to be 1.92 and 1.70 , respectively. CS was formed with a relatively uniform lignin fragment size and a lower number of condensed linkages that compared to PBL and FR.

Table 2. Average molecular weight and distribution coefficient $\left(\mathrm{M}_{\mathrm{w}} / \mathrm{M}_{\mathrm{n}}\right)$ of lignin from EL, $\mathrm{FR}$ and PBL.

\begin{tabular}{lccc}
\hline Lignin & $\overline{\mathrm{M}_{\mathrm{W}}}$ & $\overline{\mathrm{M}_{\mathrm{n}}}$ & $\mathrm{D}$ \\
\hline $\mathrm{CS}$ & 3660 & 1903 & 1.92 \\
$\mathrm{FR}$ & 6416 & 3762 & 1.70 \\
PBL & 5520 & 3958 & 1.40 \\
\hline
\end{tabular}

FTIR analysis. The spectra of FTIR analysis involved most of lignin's characteristic bands are shown in Fig. 1. The major peaks were the absorption at $3408 \mathrm{~cm}^{-1}$ due to O-H stretching vibration, $2932 \mathrm{~cm}^{-1}$ originated from C-H stretching in methoxyl and methylene groups, $1702 \mathrm{~cm}^{-1}$ arised from unconjugated carbonyl stretches. $1603 \mathrm{~cm}^{-1}, 1507 \mathrm{~cm}^{-1}$ and $1419 \mathrm{~cm}^{-1}$ for aromatic skeletal vibration.

In the spectra of lignin products, the syringyl ring breathing at $1323 \mathrm{~cm}^{-1}$ was much more weak than the band at $1211 \mathrm{~cm}^{-1}$ (guaiacyl ring breathing with C-O stretching) [4], which indicates that the obtained lignin predominantly consisted of guaiacyl units. Compared the FTIR spectra, it was clear that the strong band at $1028 \mathrm{~cm}^{-1}$ (C-O stretching vibration) almost disappeared in those lignin, which means that carbohydrates were not exsisted. 


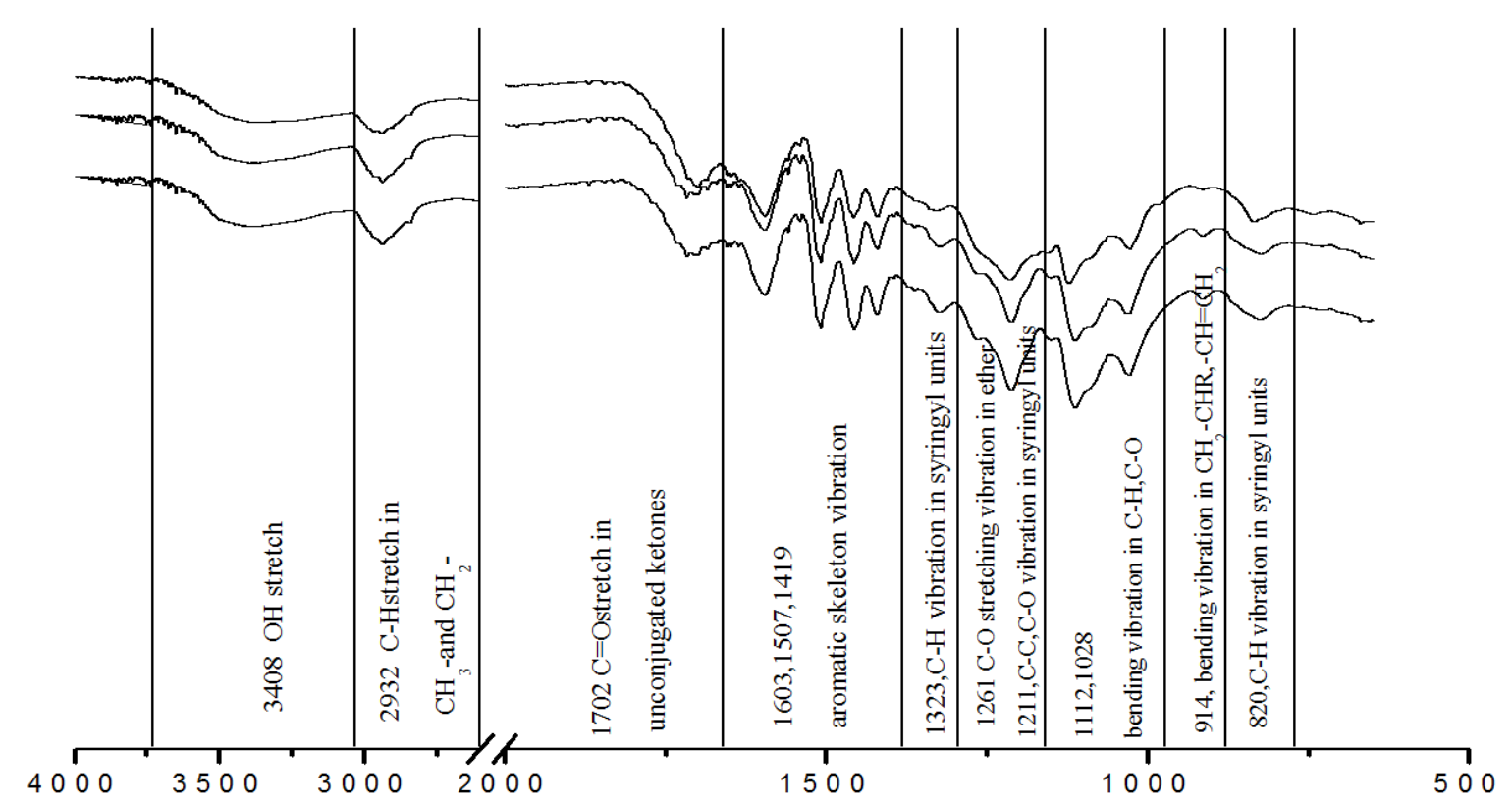

Figure 1. FT-IR spectra of enzymatic hydrolysis lignin from the corn stalks (CS) and furfural residue (FR)

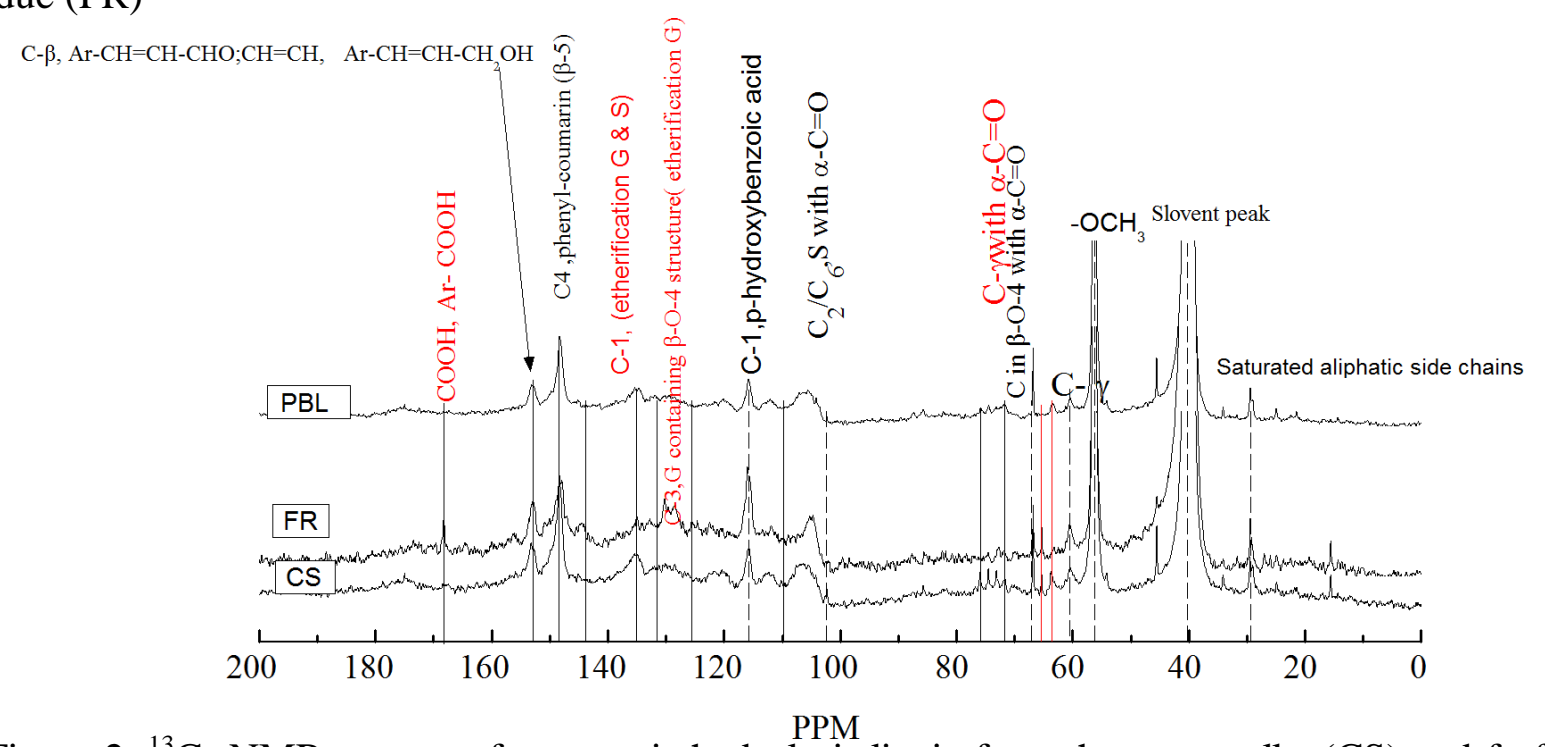

Figure 2. ${ }^{13} \mathrm{C}$-NMR spectra of enzymatic hydrolysis lignin from the corn stalks (CS) and furfural residue (FR)

${ }^{13} \mathbf{C}$ - and ${ }^{1} \mathbf{H}$ - NMR analysis. In the ${ }^{13} \mathrm{C}$-NMR spectra, the signals at $40 \mathrm{ppm}$ arose from DMSO- $\mathrm{d}_{6}$. Most of the lignin signals, such as the methoxyl carbon was shown at 55.5 to $56 \mathrm{ppm}$ [4]. However, the signal at 62-63 ppm was not observed in PBL. This signal is assigned to the $\mathrm{C}-\gamma$ with $\alpha-\mathrm{C}=\mathrm{O}$ originated from side chain oxidation via hemolytic cleavage during enzymatic hydrolysis process. The signal at 72 ppm were attributed to $\mathrm{C} \alpha$ in $\beta-\mathrm{O}-4$, those of CS and FR were intense than PBL, which indicates that the lignin of CS and FR consisted of $\beta$-O-4units. The signal at $167 \mathrm{ppm}$ of FR was $\mathrm{C}=\mathrm{O}$ in carboxyl. Furthermore, a few differences are seen only in the ${ }^{1} \mathrm{H}-\mathrm{NMR}$ spectra, the signals at $2.5 \mathrm{ppm}$ arose from DMSO-d6. The resonance of aliphatic and aromatic acetyl groups were shown at 1.9 and $2.2 \mathrm{ppm}$ respectively [4]. Signal for methoxyl groups was observed in the region between 3.5 and $4.0 \mathrm{ppm}$. The signals from 6.5 to $7.4 \mathrm{ppm}$ were attributed to the aromatic protons, in which $6.5-6.8 \mathrm{ppm}$ was assigned to the aromatic protons of the syringyl units $(\mathrm{S})$ and 6.8-7.4 ppm was corresponding to guaiacol units $(G)$ [3]. In particular, the signal at $3.2 \mathrm{ppm}$ is normally assigned to the $\mathrm{H}_{\beta}$ in $\beta-\beta$ structures. As mentioned above for Fig. 3, reduction of the signal intensity at $3.2 \mathrm{ppm}$ is also good evidence for condensation of $\beta-\beta$ structures during FR hydrolysis. The higher Mw value for FR is good evidence for FRcondensation by production process. The results divulge that the lignin products separated from residue had fairly potential application with greater chemical activity. 


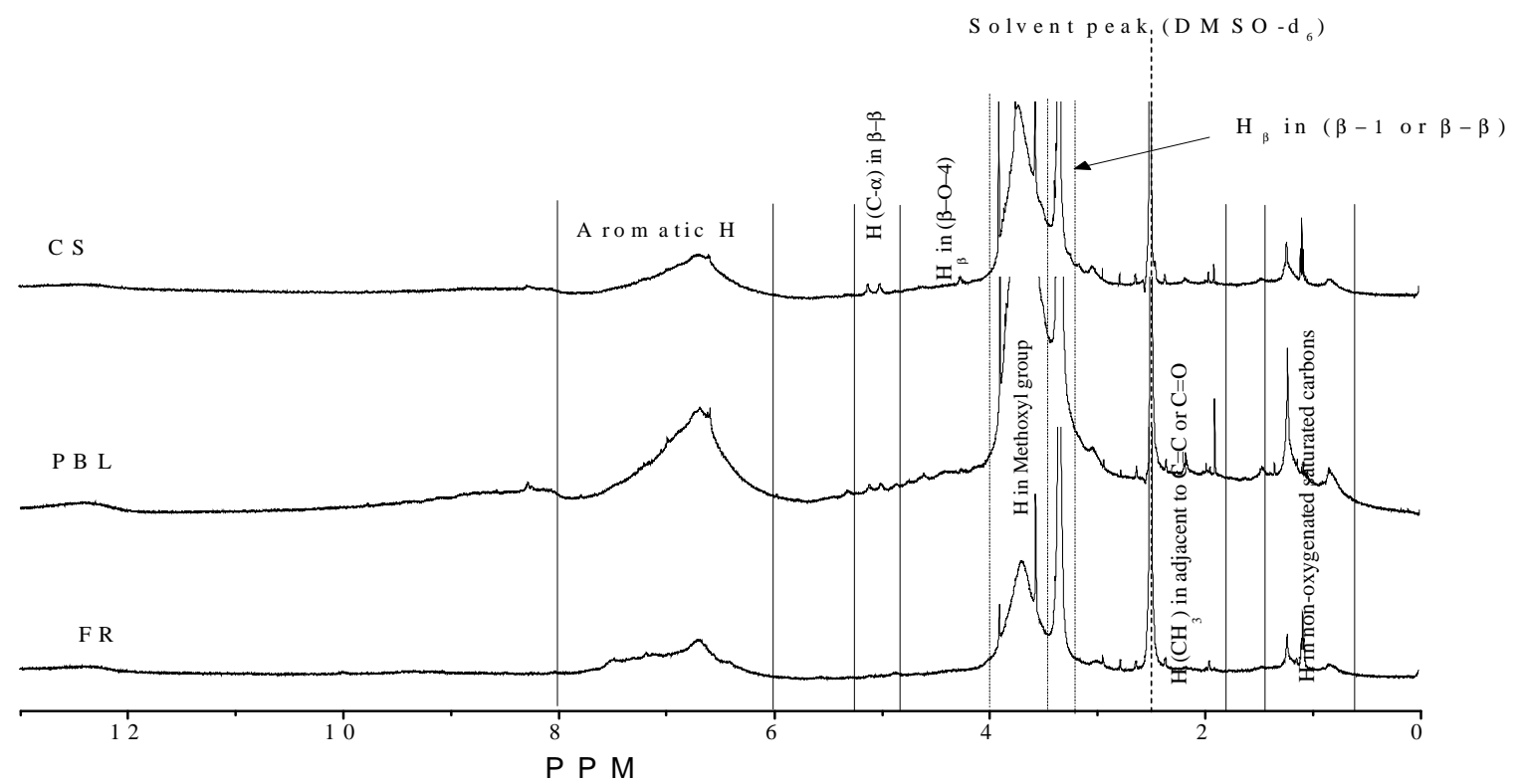

Figure 3. ${ }^{1} \mathrm{H}-\mathrm{NMR}$ spectra of enzymatic hydrolysis lignin from the corn stalks (CS) and furfural residue (FR)

\section{Conclusions}

In this study, structural features of enzymatic hydrolysis lignin from the corn stalks and furfural residue was investigated in batch systems. CS and FR consist of more free-phenolic moieties that are probably released by partial fragmentation of lignin during the enzymatic hydrolysis extraction. CS was formed with a relatively uniform lignin fragment size and a lower number of condensed linkages that compared to PBL and FR. Enzymatic hydrolysis lignin from the corn stalks (CS) and furfural residue (FR) were not exist carbohydrates. The lignin of CS and FR consisted of $\beta$-O-4units, the $\mathrm{C}-\gamma$ with $\alpha-\mathrm{C}=\mathrm{O}$ originated from side chain oxidation via hemolytic cleavage and condensation of $\beta-\beta$ structures during enzymatic hydrolysis process.

\section{Acknowledgements}

This work was supported by the Fundamental Research Funds for the Central Universities (No. DL13CB09), the Youth Science Foundation of Heilongjiang Province of China (No. QC2014C011), Program on College Students' Innovation Experiment (201410225095).

\section{Preferences}

[1] Fenfen Guo, Wenjing Shi, Wan Sun, Xuezhi Li, Feifei Wang, Jian Zhao and Yinbo Qu, Differences in the adsorption of enzymes onto lignins from diverse types of lignocellulosic biomass and the underlying mechanism, Biotechnology for Biofuels, 2014, 7:38.

[2] Guowan Guo, Shujun Li, Lu Wang, Shixue Ren, Guizhen Fang, Separation and characterization of lignin from bio-ethanol production residue. Bioresource Technology, 2013, 135: 738-741.

[3] Jae-Young Kim, Eun-Jin Shin, In-Yong Eom, Keehoon Won, Yong Hwan Kim, Donha Choi, In-Gyu Choi, Joon Weon Cho, Structural features of lignin macromolecules extracted with ionic liquid from poplar wood, Bioresource Technology, 2011, 102(19): 9020-9025.

[4] Ming-Fei Li, Shao-Ni Sun, Feng Xu, Run-Cang Sun. Sequential solvent fractionation of heterogeneous bamboo organosolv lignin for value-added application, Separation and purification technology, 2013, 101(13): 18-25. 